\title{
Review
}

\section{An Overview of Traditional Chinese Herbal Formulae and a Proposal of a New Code System for Expressing the Formula Titles}

\section{Yeong-Deug Yi and II-Moo Chang}

\author{
Natural Products Research Institute, Seoul National University, Seoul, Korea
}

\begin{abstract}
Traditional Chinese herbal therapy can be characterized by the use of a large number of multi-herb formulae. To provide modern and Western scientists without knowledge of Chinese literature and cultural background easy access to information, a database with a total of 11810 traditional Chinese herbal formulae was constructed. All the information was then translated into understandable scientific terms in English. While coining the formula titles in English, we discovered some principles governing the naming of titles by using computer analysis. In addition, we observed that about $92 \%$ of the formulae are in the range of single-herb formulae to thirteen-herb formulae. Most large number-herb formulae are formulated by combining pre-existing smaller number-herb formulae. The King herbs (君藥) with major therapeutic activity in a multi-herb formula were identified by the formulation concept using two parameters: the herbal dose and the herbal drug property (the degree of toxicity). Based on such analytical data, we established an English code system representing all formula titles written in ideographic Chinese characters: an array of important key words such as 'Herbal name in Latin + Efficacy (Target organs) + Preparation form + Number of herbs.' By searching the English version of the database with any of the above key words, a variety of information on the status of traditional Chinese herbal therapy can be accessed.
\end{abstract}

Keywords: traditional Chinese herbal therapy - herbal database - code system - formula title

\section{Introduction}

When researchers, especially Western scientists without knowledge of Chinese language and cultural background, attempt to access information on traditional Chinese medicine, they encounter a large number of medical literatures and classics. According to an opinion on world medical history, traditional Chinese medicine was one of the advanced medical sciences until the late 17 th century. A large number of medical literatures and classics have been recorded; however, more than 12000 medical classics are still scattered in libraries throughout China (1). Numerous surgical operations were actively performed, and even eye surgery was conducted using small apparatuses (2). However, presently, only acupuncture and

For reprints and all correspondence: Il-Moo Chang, Natural Products

Research Institute, Seoul National University, Yungun-dong,

Jongro-ku, Seoul 110-460, Korea. Fax: +82-2-745-1015.

E-mail: changim@snu.ac.kr herbal drug therapies are practiced as major branches of traditional Chinese medicine (TCM). These two therapies tend to draw research interests in the fields of Complementary and Alternative Medicine (CAM); moreover, acupuncturist and herbalist licenses are legally issued in several Western countries. Unlike other traditional herbal therapies across the world, traditional Chinese herbal therapy is characterized by the use of a large number of multi-herb formulae (the combination of several herbs in a single formula). A recent publication based on medical classics lists nearly 100000 multi-herb formulae (3). Endeavors to manually read and memorize such information may require inordinate efforts. Perhaps, it is virtually impossible to understand the profound wisdom of traditional Chinese herbal therapy. To deal with such a large number of herbal formulae and their contents, it is desirable to construct a database (DB) and to provide an accurate translation of the formulae in English for Western scientists. Otherwise, the paradigm of bridging the gap between modern and traditional

The online version of this article has been published under an open access model. Users are entitled to use, reproduce, disseminate, or display the open access version of this article provided that: the original authorship is properly and fully attributed; the Journal and Oxford University Press are attributed as the original place of publication with the correct citation details given; if an article is subsequently reproduced or disseminated not in its entirety but only in part or as a derivative work this must be clearly indicated. 
medicines would be difficult to achieve. In our laboratory, we have constructed a database of traditional Chinese herbal therapy, namely the TradiMed DB (4-6) in past 10 years. During the construction of the English version, we faced two major obstacles. The first was the issue of the method for translating the titles of the formulae into English; the second was the issue of expressing the traditional terminology of diseases and symptoms in corresponding modern pathological terms. To solve the first problem, we analyzed traditional formulae by using the TradiMed DB.

\section{Distribution patterns of multi-herb formulae}

The TradiMed DB contains a total of 11810 formulae that were extracted from 13 medical classics published in China and Korea. Selection of medical classics was based on their time-periods and their importance in TCM history. The ShangHan-Lun (傷寒論, 張仲景) of the Han (漢代) dynasty, the DanXi-Yi-Ji (丹溪醫集, 朱震亨) of the Yuan (元代) dynasty of China, and the Dong-Eui-Bo-Gam (東醫寶監, 許俊) of the Chosun (朝鮮) dynasty of Korea (same period as the Ming dynasty of China) were major resources. The Dong-Eui-BoGam lists more than 6800 formulae extracted from 86 medical classics written during the period between the Han dynasty and the early Ming dynasty. Of the total 86 books referred, 83 were Chinese medical classics and three were Korean. An additional 10 Korean medical classics that were mostly revised versions of the Dong-Eui-Bo-Gam were also referred to.
A total of 3196 herbal materials were used to constitute 11810 formulae. On classifying several different species belonging to the same genus of medicinal plants as one plant, the number was reduced to 839 medicinal plants (for example, Glycyrrhiza uralensis and G. glabra are considered as one herb-Glycyrrhizae radix; the same rule was applied to processed herbs as well; for example, roasted Glycyrrhizae radix was classified as Glycyrrhizae radix). The total number of 11810 formulae were reduced to 6986 by the combination of 839 herbal materials. The distribution pattern by the number of multi-herbs in a formula is shown in Fig. 1. About $92 \%$ of the total formulae are distributed in the range of single-herb formulae to thirteen-herb formulae. In addition, about $91 \%$ of the total formulae (6986) are multi-herb formulae. It is also noted that the number of multi-herb formulae having more than 17 herbs have rapidly reduced. Therefore, it is reasonable to assume that single herbs were more commonly used as herbal remedies for human ailments in ancient times. Later, in the long course of adaptation and development, multi-herb formulae were introduced by combining the pre-existing multi-herb formulae. In fact, Juzen-taiho-to, a multi-herb formula studied in Yamada's paper (7) in this journal, consists of 10 herbal materials with two different multi-herb formulae (each formula consists of four herbal materials) and two auxiliary herbs.

It should be mentioned that the term 'herbal materials' used in this paper actually includes medicinal plants, animal materials and minerals. Therefore, we examined the manner in which they were used to comprise the single- and the multi-herb

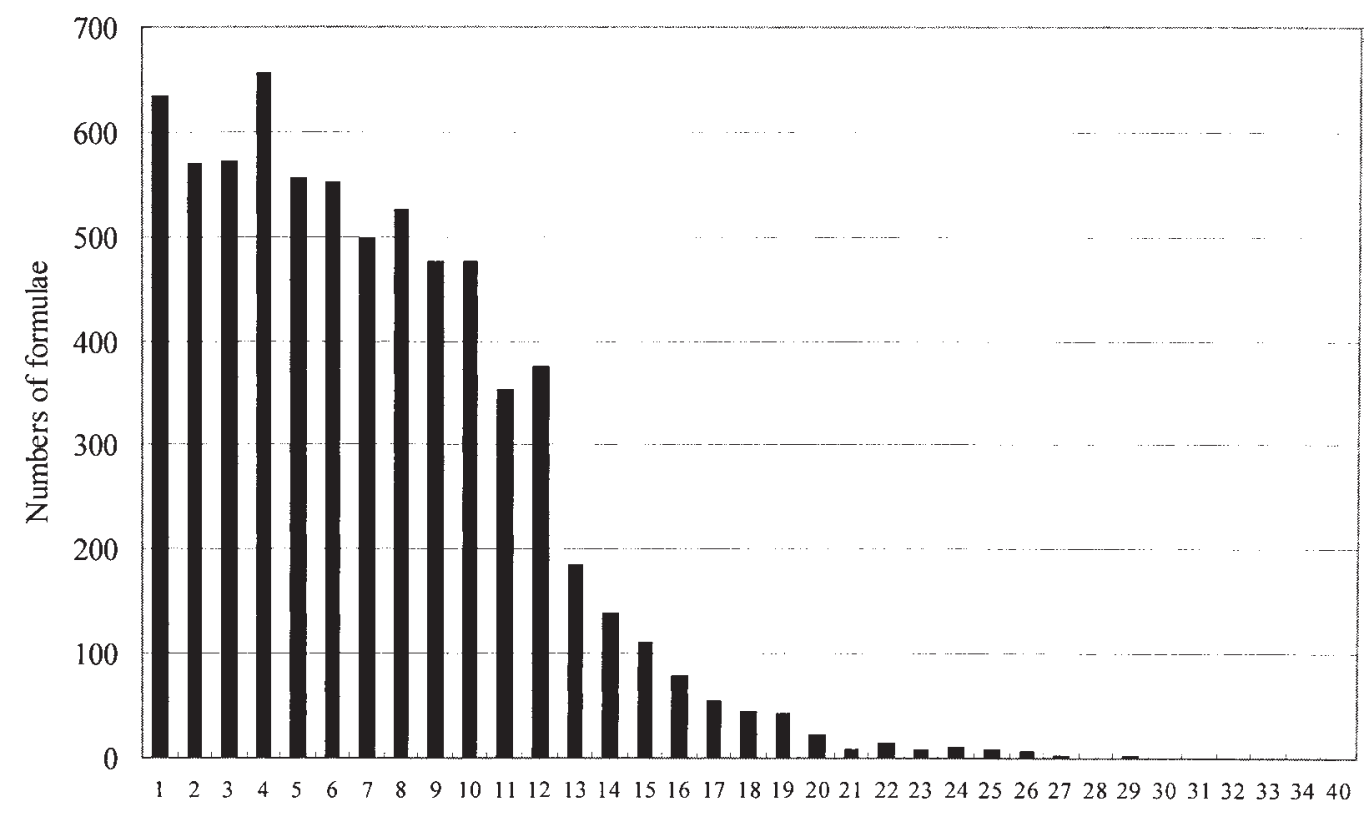

Numbers of constituting herbs

Figure 1. Distribution of TCM formulae by the number of constituting herbal materials. Number of constituting herbs:single-herb formulae (635); 2-herbs multi-formulae (570); 3-herbs (571); 4-herbs (655); 5-herbs (556); 6-herbs (551); 7-herbs (498); 8-herbs (526); 9-herbs (476); 10-herbs (475); 11-herbs (354); 12-herbs (375); 13-herbs (184); 14-herbs (139); 15-herbs (110); 16-herbs (79); 17-herbs (55); 18-herbs (45); 19-herbs (42); 20-herbs (22); 21-herbs (9); 22-herbs (14); 23-herbs (9); 24-herbs (10); 25-herbs (8); 26-herbs (6); 27-herbs (3); 28-herbs (1); 29-herbs (2); 30-herbs (1); 31-herbs (1); 32-herbs (1); 33-herbs (1); 34-herbs (1); 40-herbs (1). 
formulae as shown in Fig. 2. Of the 839 herbal materials, 467 were plant materials, 293 were animal materials and 79 were minerals $(56 \%, 35 \%$ and $9 \%$, respectively). The data showed that a variety of animal products were used. Of the 839 herbal materials, 204 were used only for multi-herb formulae, 159 were used only for single-herb formulae, and the remaining 476 were used for both single- and multi-herb formulae. Thus, the total number of single-herb formulae is $635(159+476)$. It is worth mentioning that among the 159 single-herb formulae, animal materials (102) were more commonly used than plant materials (49). One of the possible reasons for this is that many of the single-herb formulae consisting of animal materials are used as tonic foods, which can be equated to dietary supplements in modern terms. Further analysis on the formulation in relation to the kinds of materials was preformed and summarized as shown in Table 1. Of the 6351 multi-herb formulae, 3609 consist of only plant materials (57\%), 1052 are derived from a combination of plant and animal materials (17\%), 948 use plants and minerals (15\%) and 556 are derived from all the three sources (9\%). A small number of formulae consist of only animal materials or only minerals or both animal materials and minerals. The data shows that TCM uses a relatively large number of animal or mineral materials along with plant materials

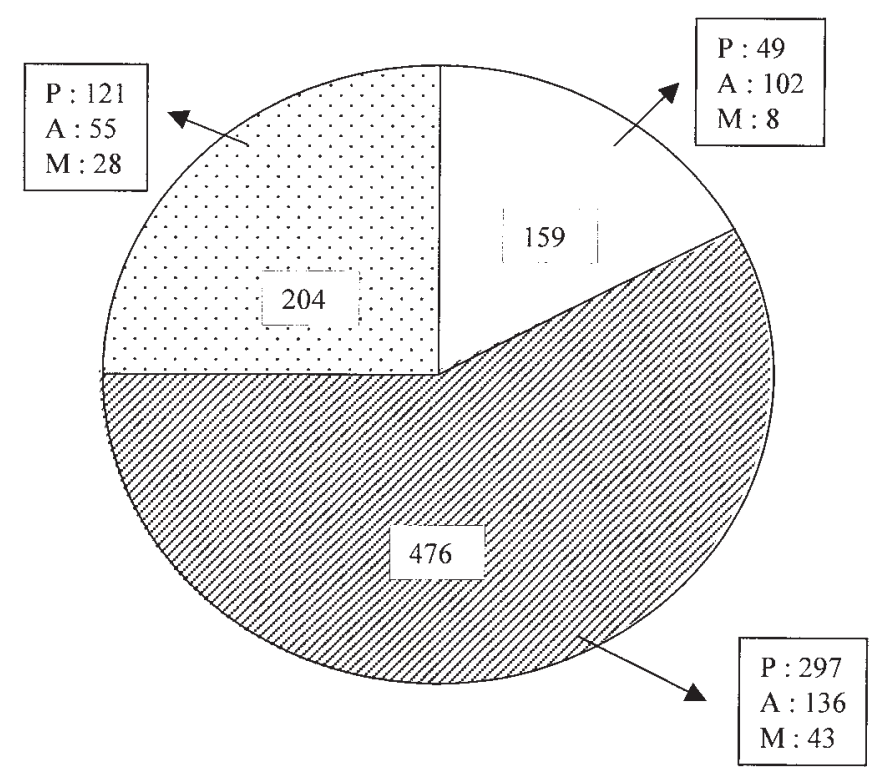

Figure 2. Types of herbal materials and distribution in the single- and multiherb formulae. P: Plant materials; A: Animal materials; M: Mineral materials. "Unfilled area: 159 herbal materials used for only single-herb formulae; dotted area: 204 herbal materials used only for multi-herb formulae; striped area: 476 herbal materials used for both single-herb and multi-herb formulae". for formulation. In addition, the results shown in Fig. 2 and Table 1 indicate that only 680 herbal materials $(204+476)$ obtained from plant, animal and mineral sources are used to create 6351 multi-herb formulae through various combinations according to the unique traditional medical doctrine (a type of grand hypothesis of Yin-Yang and Five Elements theory). Herbal therapy is expected to exhibit multi-organ and/or holistic effectiveness using such multi-herb formulae.

\section{How can new multi-herb formulae be developed?}

In relation to the use of herbal drugs in human history, both Oriental and Occidental worlds applied the principle of 'Similia similibus' to the selection of plant and animal materials. It is believed that single herbs were predominantly used as remedies for human ailments since ancient times. Along with selecting reliable herbal drugs based on clinical experiences, it is reasonable to assume that the single-herb formulae were verified with certain efficacies. Further, the multi-herb formulae were developed gradually by adding one or more herbs to the pre-existing single-herb formulae. Thus, even as much as forty herbs have appeared in a multi-herb formula in the field of traditional Chinese herbal therapy (Fig. 1). It has been interesting to investigate whether a new multi-formula is obtained just by adding a certain herbal material or by combining two or more pre-existing multi-herb formulae. To examine this presumption, we analyzed the formulae consisting of three to sixteen herbs. The total number of multi-herb formulae is 5549 as shown in Table 2. With respect to three-herb formulae, they could be formulated in two possible ways: 1) one herb + one herb + one herb and 2) a pre-existing two-herb formula + one herb. Categories 1 and 2 are defined as "a multi-herb formula containing a new combination of single herbs, not derived from a combination of pre-existing multi-herb formulae" and "a multi-herb formula is derived from a combination of preexisting muti-herb formulae." respectively. In this manner, we examined a total of 5549 multi-herb formulae. Of the 571 three-herb formulae, 265 (46.4\%) belong to category 2, and 306 fall under category 1 . As the number of herbs constituting the multi-herb formulae increases, the number of formulae falling under category 1 is drastically reduced, whereas the number under category 2 is increased. In the case of sevenherb formulae, category 2 includes more than $88.6 \%$ formulae, indicating that most formulae are derived from a combination of pre-existing small number-herb formulae. Juzen-taiho-to, a ten-herb formula, is an example of the combination of two different four-herb formulae with two minor functional herbs. This observation strongly implies that most of the traditional

Table 1. Distribution of types of herbal materials in the single- and multi-herb formulae

\begin{tabular}{|c|c|c|c|c|c|c|c|c|}
\hline & Total & Plant(s) only & Animal(s) only & Mineral(s) only & $\begin{array}{l}\text { Plants and } \\
\text { animals }\end{array}$ & $\begin{array}{l}\text { Plants and } \\
\text { minerals }\end{array}$ & $\begin{array}{l}\text { Animals } \\
\text { and minerals }\end{array}$ & $\begin{array}{l}\text { Plants, animals } \\
\text { and minerals }\end{array}$ \\
\hline Single-herb formulae & 635 & 346 & 238 & 51 & & & & \\
\hline Multi-herb formulae & 6351 & 3609 & 55 & 56 & 1052 & 948 & 75 & 556 \\
\hline Total & 6986 & 3955 & 293 & 107 & 1052 & 948 & 75 & 556 \\
\hline
\end{tabular}


Table 2. Comparison between formulae derived from pre-existing formulae and completely new formula not derived from the pre-existing formulae

\begin{tabular}{lccr}
\hline $\begin{array}{l}\text { Number of herbs } \\
\text { in a multi-herb } \\
\text { formula }\end{array}$ & $\begin{array}{l}\text { Number of formulae } \\
\text { derived from pre-existing } \\
\text { formulae (Ratio) }\end{array}$ & $\begin{array}{l}\text { Number of new } \\
\text { formulae (Ratio) }\end{array}$ & Total \\
\hline 3 & $265(46.4 \%)$ & $306(53.6 \%)$ & 571 \\
4 & $412(62.9 \%)$ & $243(37.1 \%)$ & 655 \\
5 & $415(74.6 \%)$ & $141(25.4 \%)$ & 556 \\
6 & $461(83.7 \%)$ & $90(16.3 \%)$ & 551 \\
7 & $441(88.6 \%)$ & $57(11.4 \%)$ & 498 \\
8 & $494(93.9 \%)$ & $32(6.1 \%)$ & 526 \\
9 & $453(95.2 \%)$ & $23(4.8 \%)$ & 476 \\
10 & $453(95.4 \%)$ & $22(4.6 \%)$ & 475 \\
11 & $346(97.7 \%)$ & $8(2.3 \%)$ & 354 \\
12 & $370(98.7 \%)$ & $5(1.3 \%)$ & 375 \\
13 & $183(99.5 \%)$ & $1(0.5 \%)$ & 184 \\
14 & $138(99.3 \%)$ & $1(0.7 \%)$ & 139 \\
15 & $109(99.1 \%)$ & $1(0.9 \%)$ & 110 \\
16 & $79(100.0 \%)$ & $0(0.0 \%)$ & 79 \\
Total & $4619(83.2 \%)$ & $93(16.8 \%)$ & 5549 \\
\hline
\end{tabular}

Chinese herbal formulae with higher number of constituting herbs were formulated by combining pre-existing formulae, and very few entirely new formulae (category 1 ) were developed with seven herbs or more.

\section{Principle governing the formulation of multi-herb formulae}

When a single herb is used as a remedy for a certain ailment, it is considered that the single herb possesses a major pharmacological activity. While adding one more herb to the single-herb formula to construct a two-herb formula, it is necessary to verify the additional herb's functionality. When the number of constituting herbs is increased to three or more, identification of the herb that plays a major role among others becomes very complicated. According to the traditional principles governing multi-herb formulation, each of the constituting herbs in a formula is classified into any one of the four classifications: King (君), Vassal (臣), Assistant (佐) and Delivery servant (使). When a multi-herb formula is administered, each herb exhibits specific actions corresponding to its classification. The King is the herb with a major pharmacological activity; the Vassal aids the King herb's action through additive or synergistic activities, or by exhibiting another pharmacological activity in organs related to the major dysfunctional organ (for example, in hepatitis, the gall bladder, which is closely related to the liver, is usually abnormal; thus, the Vassal acts on the gall bladder). The Assistant herb usually performs a detoxifying activity. Considering that each of the herbs constituting a multi-herb formula possesses a variety of natural constituents, both beneficial and harmful, the Assistant herb acts to nullify such harmful activities. Finally, the Delivery servant herb helps transport the active components of the King and the
Vassal herbs to the target organs. Based on this principle, a multi-herb formula with large number of constituting herbs can have more than two King herbs from each formula, because it could have been formulated by combining two separate and pre-existing multi-herb formulae. Thus, it is very important to identify the King herb in multi-herb formulae while studying herbal pharmacology, especially in the context of modern pharmacology. Unfortunately, there is no objective definition or description in medical classics and literatures that can help identify the King herbs. Only some medical classics have a few records of King herbs, and authors described most of them subjectively.

\section{Parameters to identify the King herbs}

By undertaking a literature survey on medical classics (8) and examining the doses of each constituent herb in multi-herb formulae, we could reach a tentative conclusion for plausible parameters to identify the King herbs. The parameters are as follows:

1) The dose of each herb constituting a particular multiherb formula.

2) The drug property of a single herb: each single herb is classified with respect to its effectiveness versus toxicity, and is described in detail in the medical classics of 神農本草經 (Shen-Nong-Ben-Cao-Jing in Chinese) (9) and 郷藥集成方 (Hyang-Yak-Jip-Sung-Bang in Korean) (10) as follows:

a) High-leveled herbs (上藥) are herbs without any toxicity, and can be used as drugs as well as food materials. Usually, several tonic formulae consist of this class of herbs. Examples: Ginseng radix and Schizandra fructus.

b) Middle-leveled herbs (中藥) are herbs with slight toxicity and specific therapeutic effectiveness. Their use is usually restricted to tonic food materials, and they are sometimes used as food additives. Examples: Scutellariae radix, Angelica radix and Gardeniae fructus.

c) Low-leveled herbs (下藥) are herbs with high toxicity, but specific therapeutic effectiveness. Their use is highly restricted and they are not used as food sources. Special caution is exercised by practitioners of traditional medicine when prescribing these herbs. Examples: Aconiti tuber, Pinellia rhizoma and Rhei radix.

Based on the parameters mentioned above, we could identify the King herbs in multi-herb formulae as follows:

1) Herbs with the highest dose are most likely to be King herbs.

2) When the middle-leveled or low-leveled property of herbs are prescribed in a particular formula, the corresponding herbs are considered to be the King herbs, although their doses are smaller than the other constituent herbs in the multi-herb formula. 
Using these deductions, we identified the King herbs in all formulae. Subsequently, this information was used to establish a code system for titles of traditional Chinese herbal formulae.

\section{Analysis of titles of traditional Chinese herbal formulae}

All titles of TCM herbal formulae were expressed with Chinese characters. Each of the Chinese characters has a unique meaning, because they are ideographic, unlike the phonetic Roman alphabets. Almost all titles were expressed with 3 5 characters, even though few long titles having up to 18 characters also exist. When these ideographic characters are directly translated into English, no modern scientist would understand their meaning, because there are too many words that indicate not only specific herbal materials and effectiveness but also conceptual expressions based on Chinese culture and special terms used in TCM such as Fire, Moisture, Wind, Dry, Yin, Yang, several stellar positions, etc. (3). Moreover, there are too many formula titles and several different formulae having the same titles. For example, more than 200 different formulae have Ginseng Powder (人菱散) as their title.

Before translating the formula titles into English, we analyzed the naming pattern of 6351 multi-herb formula titles as shown in Table 3. In the case of the single-herb formulae, almost all titles were expressed with the herb name and their preparation form such as decoction, pill, powder, etc. Therefore, they were excluded from this analysis. The method of naming the formula titles can be classified on the basis of eight criteria. Of the total 6351 formula titles, the most abundant titles with 'Herbal Name + Preparation Form' were notified $(36.1 \%)$, followed by titles with 'Efficacy + Preparation Form' (26.3\%). When each component of the titles from group 1 to 6 were summed up, a composite expression of 'Herb Name (or Number) + Efficacy + (or Target Organs) + Preparation Form' could be deduced. Further, this array covers about $87.4 \%$ of all the titles. Based on this analysis, we developed a new coding system for expressing Chinese herbal formula titles.

Table 3. Criteria for naming the formula titles

\begin{tabular}{lc}
\hline Criteria for naming titles & $\begin{array}{l}\text { Number of formulae } \\
\text { (Ratio) }\end{array}$ \\
\hline 1) Efficacy + Target Organ + Preparation Form & $644(10.1 \%)$ \\
2) Herb Name + Efficacy + (Target Organ) + & $467(7.4 \%)$ \\
Preparation Form & $397(6.3 \%)$ \\
3) Herb Number + Preparation Form & $83(1.3 \%)$ \\
4) Herb Number + Efficacy + Preparation Form & $2290(36.1 \%)$ \\
5) Herb Name + Preparation Form & $1668(26.3 \%)$ \\
6) Efficacy + Preparation Form & $489(7.7 \%)$ \\
7) Concepts + Preparation Form* & $313(4.9 \%)$ \\
8) Others** & $6351(100.0 \%)$ \\
Total &
\end{tabular}

*Theory of Yin and Yang, Five Elements, etc.

**Names of stellar positions, famous generals, etc.

\section{The proposal for standardization of Chinese herbal formula titles in English}

Previously, the World Health Organization (Western Pacific Region) had taken the initiative to establish the standard acupoints (meridian points) nomenclature using a combination of Roman alphabets and numbers along with the phonetic expression of Chinese characters $(11,12)$. For example, a specific acupoint located on the midline of the forehead is named as MS1, where MS represents an anatomical site, and the number indicates a needle site. By employing this code system, about 360 or little more acupoints could be designated. Such a simple code system seems to be practical for acupuncturists, especially for Western practitioners, because there is a relatively small number of acupoints to be memorized. In the case of Chinese herbal formulae, the situation is much different and more difficult than that in acupuncture points. All Chinese herbal formula titles have their own meanings in Chinese, which represent particular herbs, efficacies, target organs and the number of herbs constituting a certain formula. Therefore, it is desirable to comprehend all this information as much as possible. In addition, nearly 100000 formulae are available. Thus, it would require an inordinate effort on the part of Western herbalists and scientists to memorize these formulae, which is an impossible task.

Based on our aforementioned analysis of formula titles, we propose the following code system: Herb(s) in Latin botanical name + Efficacy (or Target organs) + Preparation forms (pill, tablet, decoction, powder, etc.) + Number of herbal materials (number of constituents in a particular formula). The first part, i.e., the herb(s) are the King herbs that are identified from a multi-herb formula by the process described earlier. When the multi-herb formula consists of two or three pre-existing multiherb formulae, the number of King herbs represented in Latin botanical names is two or three. Although a multi-herb formula consists of several constituting herbal materials, only three King herbs were designated because they could be identified without overlapping with other multi-formula titles in practice. Regarding the Efficacy aspect in this code system, we chose a major pharmacological activity from a variety of indications attributed to a formula. It is common to prescribe a TCM formula for a number of symptoms and diseases. Similarly, a modern drug such as Aspirin (acetyl salicylate) is used as an anti-inflammation, antiplatelet aggregation and analgesic agent. We have observed that more than 8576 symptoms and diseases described in TCM could be classified in terms of Western medicine and could be well translated into English. These data are not shown in this paper; however, they are stored in our database.

Based on the proposal, we describe several examples as follows:

\section{Cimicifugae-Moutan-GI-Analgesics-PD (5)}

Cimicifugae-Moutan-GI-Analgesics-PD (5), 清胃散: Qing-WeiSan; Chung-Wi-San; Seii-san; phonetic expressions in Chinese, Korean and Japanese correspondingly, consists of five herbs in the formula shown in Fig. 3. Of the five herbs, Cimicifugae has 
Code : Cimicifugae-Moutan-GI-Analgesics-PD (5)

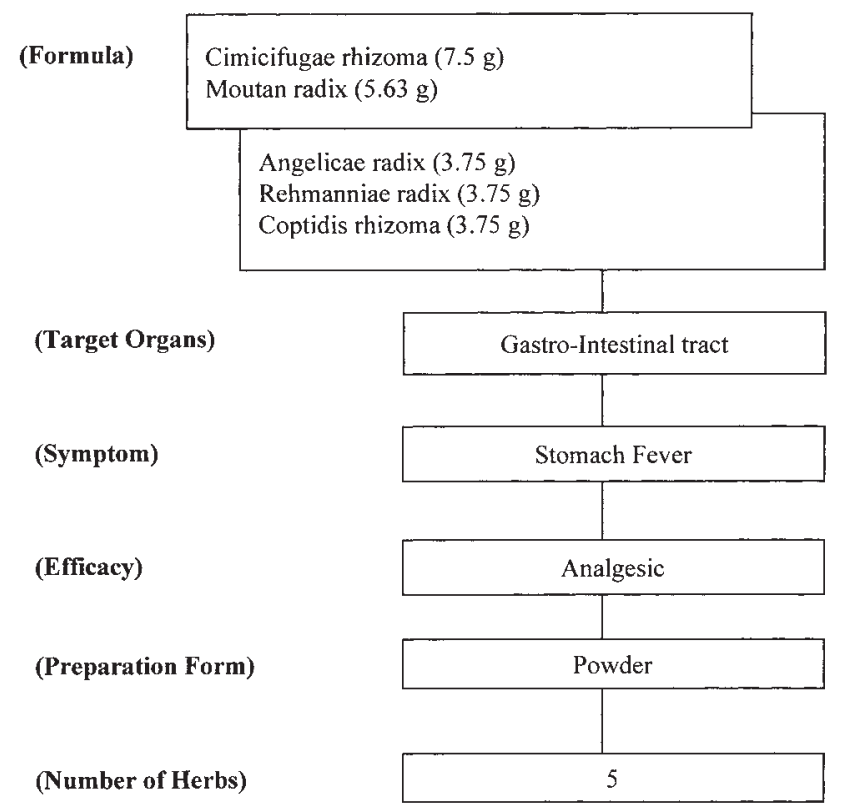

Figure 3. The code: Cimicifugae-Moutan-GI-Analgesics-PD (5): 清胃散 [Qing-Wei-San; Chung-Wi-San; Seii-san]. Cimicifugae rhizoma: 升麻 (Highleveled drug property); Moutan radix: 牧丹皮 (Middle-leveled drug property); Angelicae radix: 賞歸 (Middle-leveled drug property); Rehmanniae radix: 地黃 (High-leveled drug property); Coptidis rhizoma: 黄連 (High-leveled drug property).

the largest dose and its drug property is of the high-leveled type without any significant toxicity. Another herb with a higher dose possessing middle-leveled drug properties is Moutan radix. Therefore, the first part of the code consists of Cimifugae-Moutan in Latin expression. This formula was used for the treatment of gastro-intestinal complaints such as stomachache and heart burn. The preparation is in a powder form. The above code was constructed by combining all these key words. Similarly, three additional examples explaining the method of combining pre-existing multi-herb formulae with smaller number of herbs to make large number-herb formulae have been provided. Additionally, the method of deducing each coding title and relating them mutually has also been shown. A multi-herb formula, namely Juzen-taiho-to, consists of 10 herbs, and it is a combination of two four-herb formulae described below and two additional auxiliary herbs: one is presumably an assistant herb and another is a delivery herb as described earlier.

\section{Paeoniae-Angelicae-Blood-Antihemorrhagics-WD (4)}

Paeoniae-Angelicae-Blood-Antihemorrhagics-WD (4), 四物湯: Si-Wu-Tang; Sa-Mul-Tang; Shimotsu-to, consists of four herbs, as shown in Fig. 4 . All herbs have the same dose (4.5 g each). Paeoniae radix and Angelicae radix belong to the middleleveled drug property category, whereas the remaining two herbs are considered to be in the high-leveled drug property category. Therefore, Paeoniae and Angelicae are selected as King
Code : Paeoniae-Angelicae-Blood-Antihemorrhagics-WD (4)

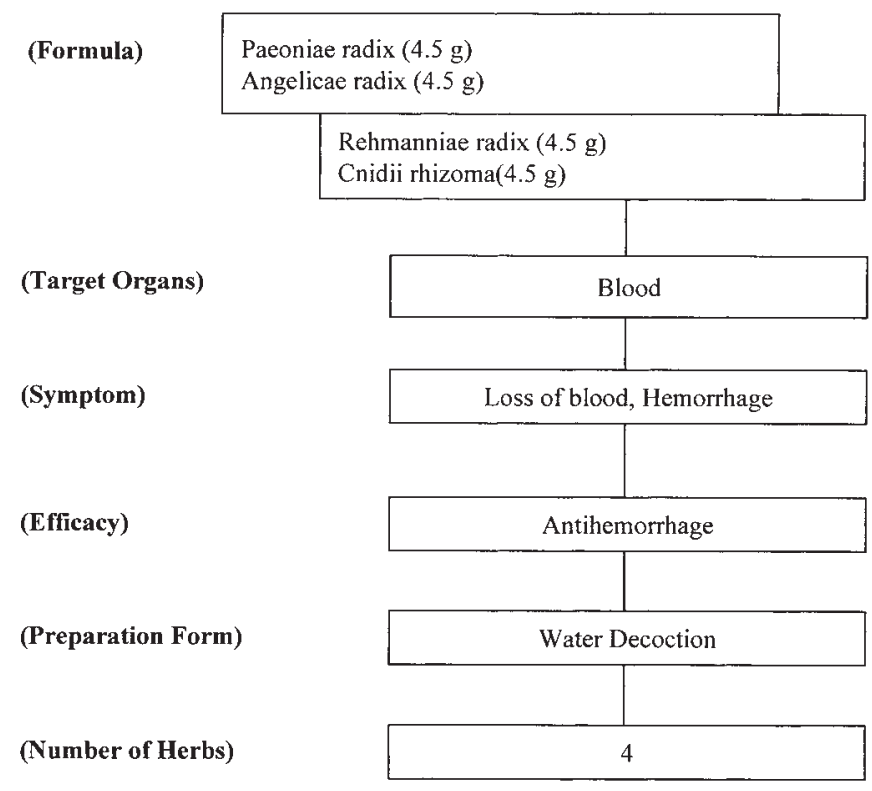

Figure 4. The code: Paeoniae-Angelicae-Blood-Antihemorrhagics-WD (4) 四物湯 [Si-Wu-Tang; Sa-Mul-Tang; Shimotsu-to]. Paeoniae radix: 茢藥 (Middleleveled drug property); Angelicae radix: 虽毁 (Middle-leveled drug property); Rehmanniae radix: 地黄 (High-leveled drug property); Cnidii rhizoma: 川节 (High-leveled drug property).

herbs. This formula has most frequently been used for various dysfunctions related to the blood, and is especially prescribed in the case of blood loss and abnormal circulation. WD stands for water decoction preparation. The number (4) indicates that this formula consists of four different herbal materials.

\section{Atractylodis-Poria-Consumptive-Tonics-WD (4)}

Atractylodis-Poria-Consumptive-Tonics-WD (4)，四君子湯: Si-Jun-Zi-Tang; Sa-Kun-Ja-Tang; Shikunshi-to, consists of four herbs as shown in Fig. 5. Of the four herbs, each herb has the same dose, and Atractylodis rhizoma and Poria belong to the high-leveled drug property category. Thus, these two are selected as King herbs. This formula has been widely prescribed for general weakness, especially during convalescence after consumptive diseases. Thus, it is classified as a tonic. It is also interesting to note that most tonic formulae consist of herbs that possess a high-leveled drug property, usually in the same doses, but not the low-leveled drugs.

\section{Paeoniae-Atractylodis-Astagali-Consumptive- Tonics-WD (10)}

Paeoniae-Atractylodis-Astagali-Consumptive-Tonics-WD (10), 十全大補湯: Shi-Quan-Da-Bu-Tang; Sip-Jun-Dae-Bo-Tang; Juzen-taiho-to, is a ten-herb formula consisting of the two separate formulae described above and two additional herbs, as shown in Fig. 6. One King herb is selected from each of the 
two four-herb formulae, and the other is chosen from the two additional herbs that do not appear in these formulae. This formula has been prescribed as a tonic for promoting general health. Therefore, only the indication of consumptive disease

Code : Atractylodis-Poria-Consumptive-Tonics-WD (4)

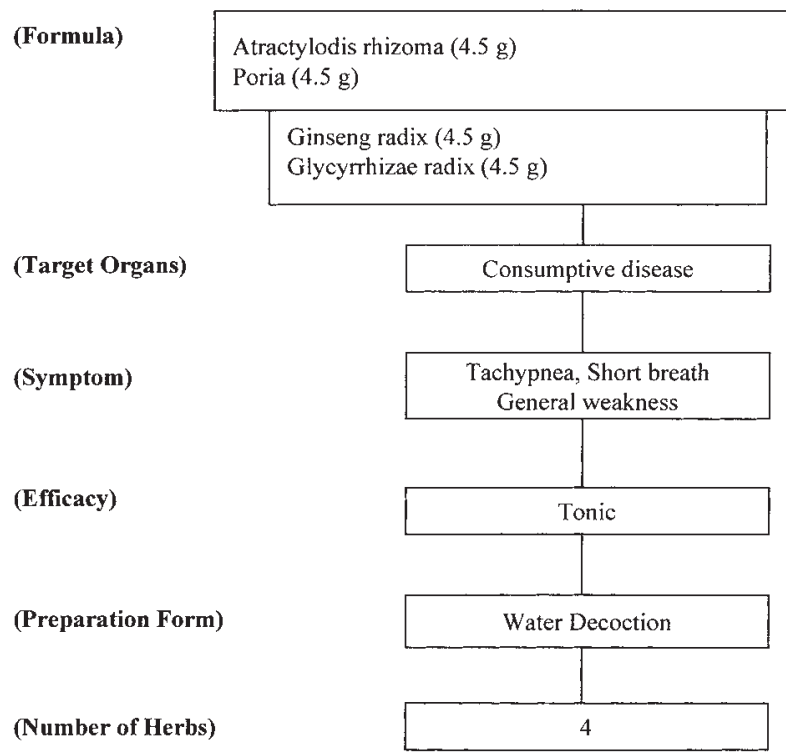

Figure 5. The code: Atractylodis-Poria-Consumptive-Tonics-WD (4): 四君子湯 [Si-Jun-Zi-Tang; Sa-Gun-Ja-Tang; Shikunshi-to]. Atractylodis rhizoma: 蒼比 (High-leveled drug property); Poria: 茯苓 (High-leveled drug property);

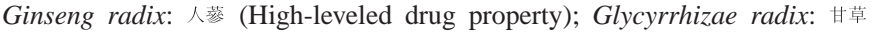
(High-leveled drug property). and the use as a tonic, and not dysfunctions related to blood, are taken as inputs to the code system.

\section{Discussion and Conclusion}

Scientists trying to access information on traditional Chinese herbal remedies, especially modern and western scientists, have faced several difficulties; these are too many multi-herb formulae written by using Chinese characters and described in terms of traditional Chinese medical doctrines. To deal with such a large number of formulae, it is desirable to construct a database in which all information is accurately translated into English. We have attempted to construct a TCM database, namely the TradiMed DB, that comprises seven groups of information: 1) a systematic botanical description of each herb with photographs of herbal materials and original plant species, 2) herbal formulae with bibliographic citations, 3) diseases and symptoms interpreted in modern medical terms (a total of 8576 till date), 4) 597 traditional processing methods used to remove toxic ingredients existing in certain herbs, 5) information on the natural constituents of Chinese herbs with chemical structures, analytical data with various spectral data and references, 6) safety and toxicity data of single herbs and formulae and 7) clinical case reports on using traditional Chinese treatments with Western medications (844 cases). In translating all this information into English, we faced two major problems: the first was the method of translating the titles of the formulae into accurate English expressions, and the second was the method of interpreting symptoms and diseases described in TCM in corresponding modern medical terms.
Code : Paeoniae-Atractylodis-Astragali-Consumptive-Tonics-WD (10)

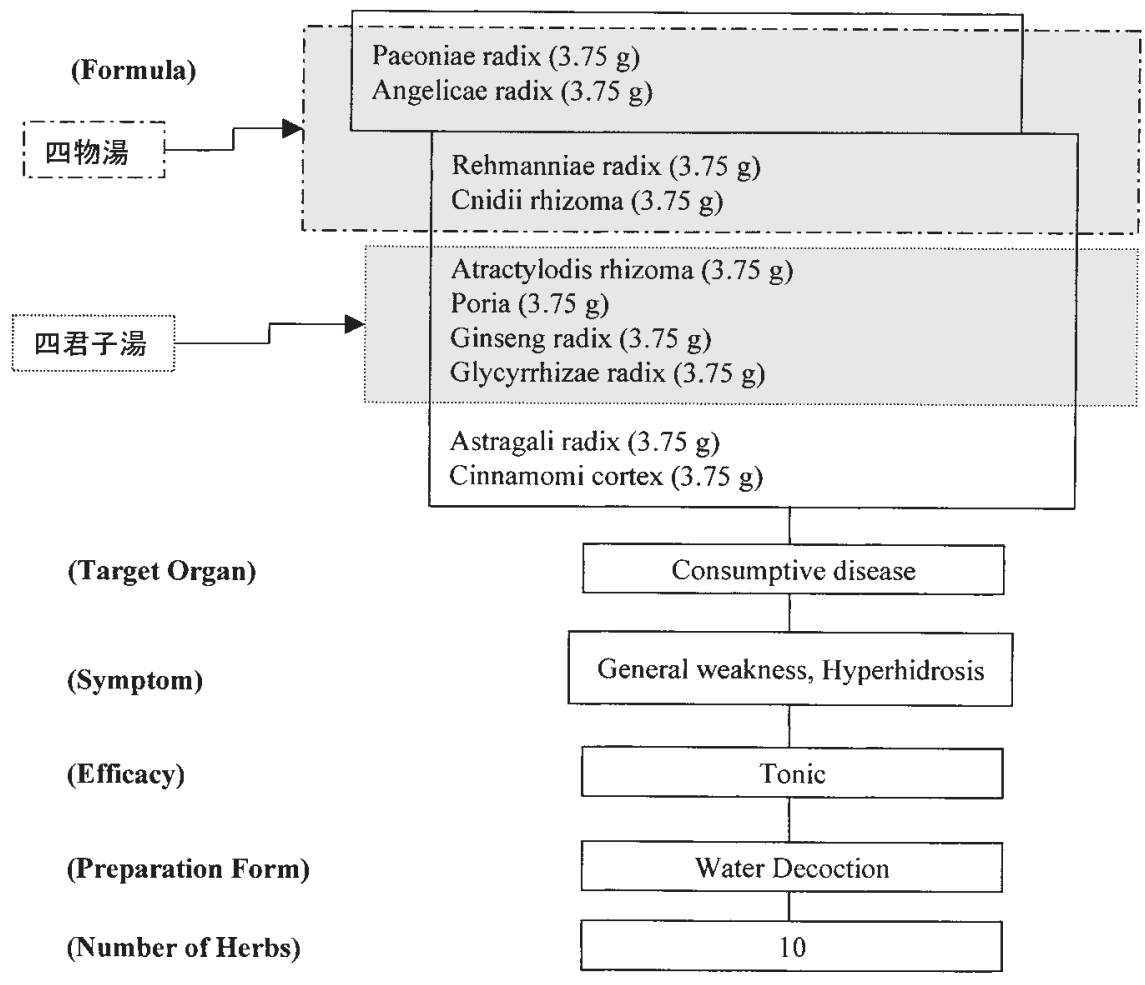

Figure 6. The code:Paeoniae-Atractylodis-AstragaliConsumptive-Tonics-WD (10): 十全大補晹 [Shi-Quan-DaBu-Tang; Sip-Jun-Dae-Bo-Tang; Juzen-taiho-to]. Paeoniae radix: 药䊾 (Middle-leveled drug property); Angelicae radix: 賞蹄 (Middle-leveled drug property); Rehmanniae radix: 地黄 (High-leveled drug property); Cnidii rhizoma: 川芦 (High-leveled drug property); Atractylodis rhizoma: 荅术 (High-leveled drug property); Poria: 获苓 (High-leveled drug property); Astragali radix: 黃蒇 (High-leveled drug property); Ginseng radix: 人落 (High-leveled drug property); Glycyrrhizae radix: 甘草 (High-leveled drug property); Cinnamomi cortex: 肉桂 (High-leveled drug property). 
In order to solve these issues, we analyzed a total of 11810 TCM formulae, and found that most multi-herb formulae, especially with a large number of constituting herbs, are formulated by combining several pre-existing formulae with a smaller number of constituting herbs. Based on such computer analysis, we developed a code system to summarize information contained in the formula titles written in Chinese. The code system summarizes the information as 'Herb(s) in Latin botanical names (the King herbs with major pharmacological activities) + Efficacy (Target organs) + Preparation form + Number of constituting herbs in a formula.'

To identify the King herb, we introduced two parameters: one was the dose and the other was the drug property of each herb, as described and used in traditional formulations. Identification of the King herb is important because it is most likely to exert a major effect. Modern scientists including Western scientists who are interested in TCM, can easily access information on traditional Chinese herbal remedies with the help of the code system, even though they may not have knowledge of Chinese culture and literature but have some basic knowledge of pharmacognosy, botany, chemistry, and diseases.

Previously, a similar attempt was made with the so-called ATC (Anatomy-Therapeutic Chemical Code) system at the WHO collaborating center for International Drug Monitoring in Uppsala, Sweden (13). This system provides some information on the therapeutic and toxic properties of traditional herbs and remedies. However, this system is not an efficient system for expressing Chinese formula titles, due to the fact that it is merely a collection of modern research findings on medicinal herbs, and can be applied only to single herbs, not to multiherb formulae.

With regard to Chinese herbal formulae, the future objective will be to study the backlog of evidence put forth by modern scientific methods. Even though three major Asian countries, China, Japan and Korea, have used the same formula titles, they are pronounced differently in all the three countries (14). Therefore, it is difficult to communicate information about them, unless all titles are written by using Chinese characters.

The code system that we propose is a prototype, and it is meant to provide a useful navigating guidance for Western scientists trying to access certain TCM formulae. Therefore, it does not cover comprehensive information on certain herbal formulae. It is desirable to develop more elaborate and refined code systems with international collaboration. However, we could express more than 10000 formulae without overlapping. Our database contains information on more than 8000 symptoms and diseases described in TCM. They could be classified in terms of Western medicine and well-translated into English.
One-third of them could be easily expressed in modern terms. Another one-third required some research involving cooperation between traditional and modern medical doctors from various fields. The remaining portion of the database needs further research for matching the symptoms or diseases with the corresponding modern medical terms. In this regard, it should be mentioned that our study on the disease-symptom classification of TCM, versus modern medicine needs further research to identify exactly matching terms. We have suggested a possible approach and a direction for the modernization of TCM which is the medical science of the 17th century. We will continue the comparative study of Chinese disease classifications versus Western terms for further refinement. Indeed, this type of study needs international efforts to bridge the gap between Eastern and Western medicines.

\section{References}

1. Xue QL. Union Catalog of Chinese Medical Literature. Beijing 1991. (全國中醫圖書聯合目錄)

2. Fu RY. Shen-Shi-Yao-Han. (1644) translated by Chang IM, Chi JG. Seoul: Natural Products Research Institute, Seoul National University: 1999, 39-41. (審視瑤函)

3. Zhang MQ. A treatise on the standardization of prescription's name. In: Chang IM, editor. Experts Meeting for the Standardization of Titles of Chinese Prescriptions. Seoul: Natural Products Research Institute, Seoul National University, WHO Collaborating Center for Traditional Medicine: 1996, 33-9. (論方制名稱的標准化)

4. Chang IM. TradiMed®: Traditional Oriental Medicine Database (http://www.tradimed.com/).

5. Chang IM, Chi JG. Harmonization of traditional oriental (Chinese) medicine and modern medicine: A step forward with TradiMed database 2000 In: Cha KY, editor. The Proceeding of Traditional and Alternative Medicine. Seoul: Pochon Cha Hospital: 1999, 151-60.

6. Chang IM. Anti-aging and health-promoting constituents derived from traditional oriental herbal remedies. Ann N Y Acad Sci 2001;928: 281-6.

7. Kiyohara H, Matsumoto T, Yamada H. Combination effect of component herbs of a Japanese herbal (Kampo) medicine, Juzen-taiho-to on expression of intestinal immune system modulating activity. in this journal.

8. Huh J. Dong-Eui-Bo-Gam. (1596) translated by Kim YH, et al. Seoul: Nam-San-Dang: 1966, 1084-141. (東醫寶鑑)

9. Shen-Nong-Ben-Cao-Jing. (6th century) revised by $\mathrm{Xu} \mathrm{SN,} \mathrm{Niu} \mathrm{BZ.}$ Heibei: Heibei Ke-Xue-Ji-Shu: 1993, 1-3. (神農本草經)

10. Kwon C, Yu HT, Rho JL, Park YD. Hyang-Yak-Jip-Sung-Bang. (1433) translated by Shin MS, et al. Seoul: Young-Lim Publishing: 1989, 1697-2086. (鄉藥集成方)

11. Standard Acupuncture Nomenclature: Part 2 Revised Edition. Manila: World Health Organization, Regional Office for the Western Pacific 1994

12. Han S. Guidelines for Clinical Research on Acupuncture. Manila: World Health Organization, Regional Office for the Western Pacific 1995.

13. Farah M. Draft Guidelines for Herbal ATC Classification. Uppsala: the Uppsala Monitoring Center, WHO Collaborating Center for International Drug Monitoring 2002.

14. Park J, Park HJ, Lee HJ, Ernst E. What's in a name? A systematic review of the nomenclature of Chinese medical formulae. Am J Chin Med 2002;30:419-27. 


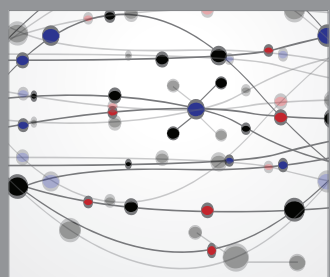

The Scientific World Journal
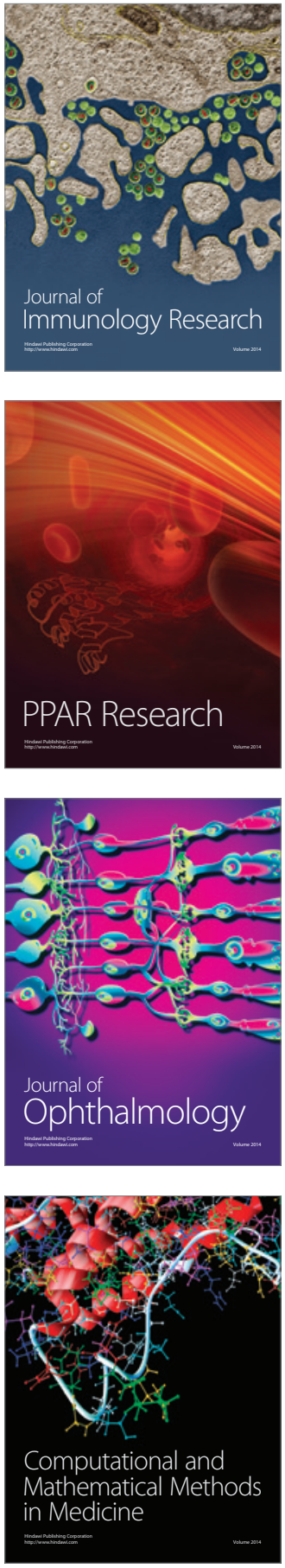

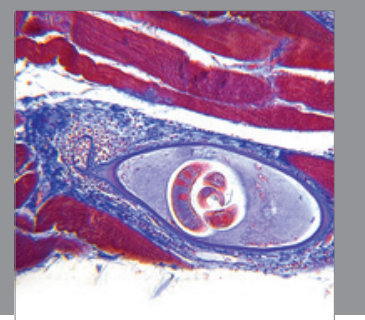

Gastroenterology

Research and Practice
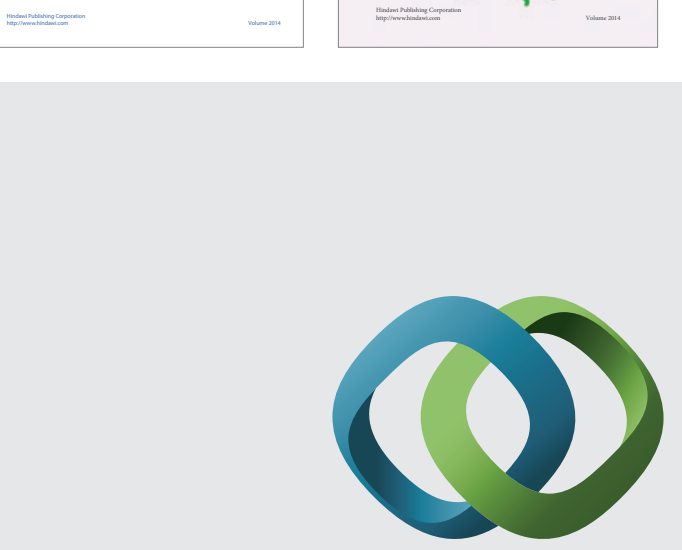

\section{Hindawi}

Submit your manuscripts at

http://www.hindawi.com
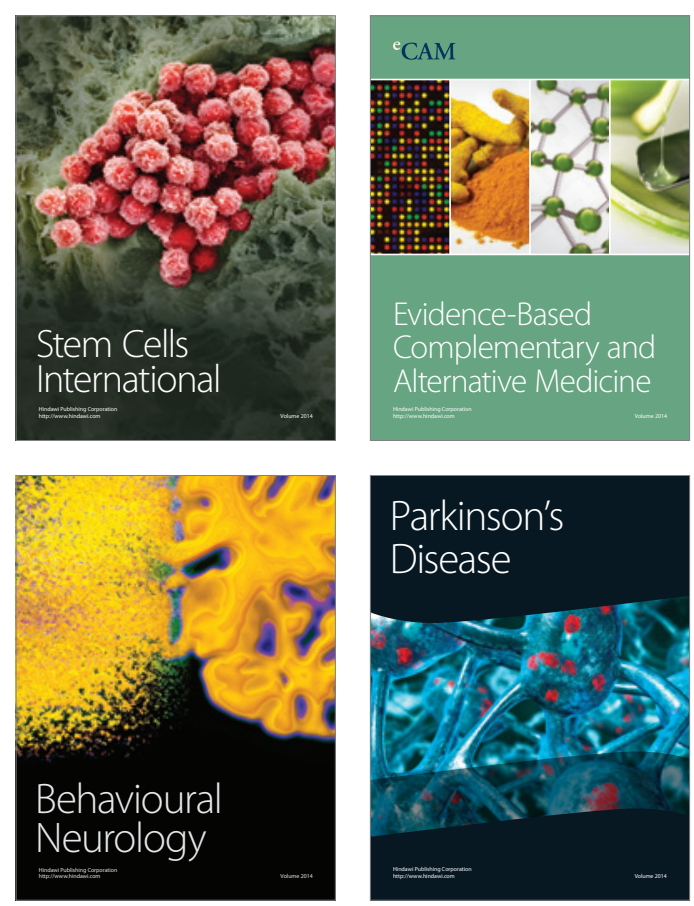

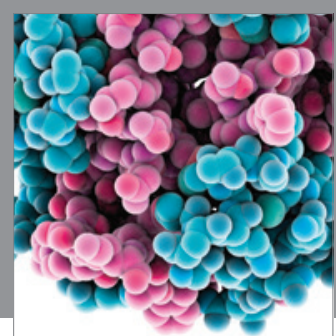

Journal of
Diabetes Research

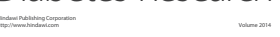

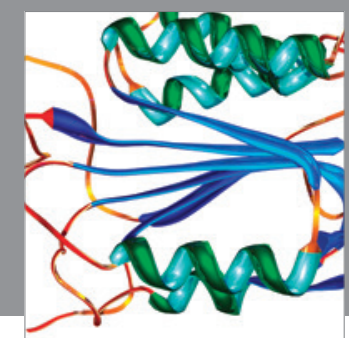

Disease Markers
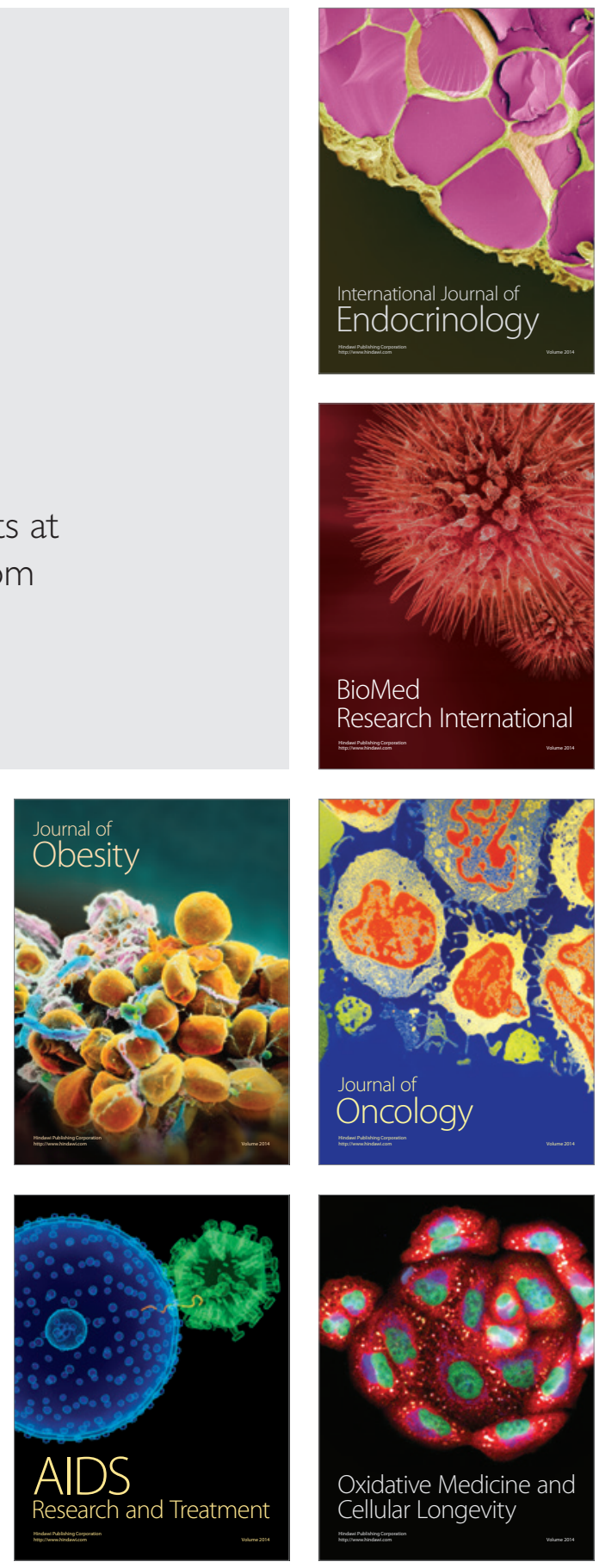